\title{
DIFFERENTIAL EMISSION MEASURE ANALYSIS OF HOT-FLARE PLASMA FROM SOLAR-MAXIMUM MISSION X-RAY DATA
}

\author{
J. Jakimiec, ${ }^{*}$ J. Sylwester, ${ }^{* *}$ J. R. Lemen, ${ }^{* * *}$ \\ R. Mewe,$\div$ R. D. Bentley, ${ }^{* * *}$ A. Fludra, ${ }^{*}$ \\ J. Schrijveri and B. Sylwester** \\ *Astronomical Institute, Wroclaw University, Wroclaw, Poland \\ ** Space Research Center, Polish Academy of Sciences, Wroclaw, \\ Poland \\ ***Mullard Space Science Laboratory, Holmbury St. Mary, \\ Dorking, England \\ † Space Research Laboratory, Utrecht, The Netherlands
}

ABSTRACT

We have investigated differential emission measure (DEM) distribution of hot flare plasma ( $>10 \mathrm{NK}$ ) using SNIV $X$-ray data from Bent Crystal spectrometer (BCS) and Hard X-ray Imaging Spectrometer (HXIS). We have found that the analysis provide a very sensitive test of consistency of observational data coming from different instruments or different channels of the same instrument. This has allowed to eliminate some systematic differences contained in the analysed data.

Typical examples of the DEM distribution are discussed. It is stressed that these improvements in the multitemperature flare diagnostics are very important for the discussion of flare energetics.

\section{INTRODUCTION}

It is well known that plasma temperature may be estimated from the intensity ratio of two $X-r a y$ features (lines or narrow spectral intervals). The estimated temperature can be called an isothermal approximation (IA) temperature. The temperatures, $T_{\mathrm{Ca}}$ and $\mathrm{T}_{\mathrm{Fi}}$, estimated from the ratios of the dielectronic satellite line ( $k$ ora $j$ ) to the resonance line (w) in the BCS calcium and iron spectra are good examples of such IA temperatures $/ 1,2 /$. When we estimate, however, the IA flare temperatures from various pairs of lines (e.g. $T_{\text {fa }}$ and $T_{F e}$ ) or various pairs of narrow $X$-ray bands (e.g. HXIS charnels), we ofiain, in general, different values of the temperature. This indicates that there is some temperature distribution in the hot flare plasma and that some information about the temperature distribution is contained in our observations. However, the problem of determination of the distribution is rather difficult.

The energy flux measured in a spectral line or a narrow $\mathrm{X}$-ray interval can be expressed as follows:

$$
F_{i}=\int I_{i}(T) \varphi(T) d T
$$

where $f_{i}(T)$ is the emission function for the spectral feature and $\varphi(T) d T=$ $\mathrm{N}^{2} \cdot \mathrm{dV}$ is the differential emission measure (DEM) distribution of the emitting plasma. If the number of measured spectral features is $k$, we have $k$ integral conditions (1) for the determination of the function $\varphi(T)$.

The main difficulties of determining the differential emission measure $\varphi(T)$ are the following: a) In present experiments the number of measured spectral features is not large $(k \leqslant 10)$ and therefore there may be many different solutiors $\varphi(T)$ which fulfil the observational conditions (1). b) Moreover, we have found that moderate systematic errors can strongly influence the obtained solution $\varphi(\mathrm{I})$. We have estimated that in order to overcome the difficulty, 
the systematic errors of the measured fluxes and the atomic data should be smaller than $20 \%$.

The multitemperature flare diagnostics is very important for the discussion of flare energetics. Our experience shows that using IA temperatures we can obtain only order-of-magnitude estimates of the total flare emission measure and next the thermal energy content.

In the present paper we investigate the hot flare plasma of the temperature $T \geqslant 10 \mathrm{NK}$ using BCS and HXIS data.

\section{BCS AND HXIS DATA ANAIYSIS}

Eirst, we investigated the determination of DEM from BCS data only, using the fluxes of the foun lines listed in Table 1. These are the same lines which are usually used to determine the IA temperatures $\mathrm{T}_{\mathrm{Ca}}$ and $\mathrm{T}_{\mathrm{F} e} e$

TABLE 1 Iist of the BCS Iines Used in DEM Analysis

\begin{tabular}{lllll}
\hline No & $(\AA)$ & Ion Trans & Excitation \\
\hline 1 & 3.1769 & Ca XIX W & E & N.B. Here E means direct electron \\
2 & 3.2057 & Ca XVIII K & DR & impact excitation, DR - excitation \\
3 & 1.8509 & Fe XXV w & E & by the dielectronio recomination. \\
4 & 1.8662 & Fe XXIV j & DR & \\
\hline
\end{tabular}

The line intensities were determined by fitting the Voigt profiles to the observed ones by means of the SPCFIT code developed at the MSSI /3/. The DEM distributions $\varphi(T)$ have been calclilated by means of iterative procedure worked out by withbroe $/ 4 /$ and adapted to the hot flare plasma analysis by Sylwester /5/. Usually we have used 150 iteration steps.

We have found that using standard BCS sensitivities, element abundarce ratio $\left(\mathrm{A}_{\mathrm{Fe}} / \mathrm{A}_{\mathrm{Ca}}=10\right)$ and ionization equilibrium curves (Shull and van steenberg $/ 6 /$ ), we $\mathrm{Fbtain}$ too large (more than 20\%) differences between the observed and model calculated $(0-C)$ line fluxes. Next, we have found that the agreement improves significantly if we introduce a constant factor $\propto$ by which we increase the calculated iron line fluxes. By apprpriate choice of the $\propto$ values the $0-c$ deviations could be reduced to the values smaller than $1 \%$.

This means that such a procedure provides a very sensitive test of internal agreement between the BCS calcium and iron line fluxes. The $\alpha$ values fall witrin the limits of 2.5-2.9 for several flares investigated. This indicates that a significant systematic error is contained in our primary data. The source of the error may be the following: a) a systematic error in the assumed sensitivities of BCS, b) an error in the assumed iron-to-calcium abundance ratio, c) a systematic error in the ionization curves used. Our preliminam analysis suggest that case $c$ is the most probable, viz. the assumed Fe XXV ion concentration is about factor 3 too low in the investigated temperature range (T*15$20 \mathrm{MK}$ ).

The $\alpha$-correction have been applied in further DEII calculations. An example of the DEN distribution derived from BCS data only is shom in Fig. 1 (solid line).

Similarly, DEM distributions have been calculated from HXIS fluxes using the emission functions published in $1983 / 7 /$. An example is shom in $F i z$. 1 (dashed line). Similar results have been obtained for many time points of several SMM flares. Therefore we have concluded that there is a systeratic shift of the HXIS temperature scale in relation to the BCS scale ( $\triangle T \approx 6$ IF for HXIS 
coarse field of view) which we have introduced into our further calculations. Moreover, a constant factor has been introduced into HXIS fluxes in order to compensate for the systematic difference in the vertical scales in Fig. 1.

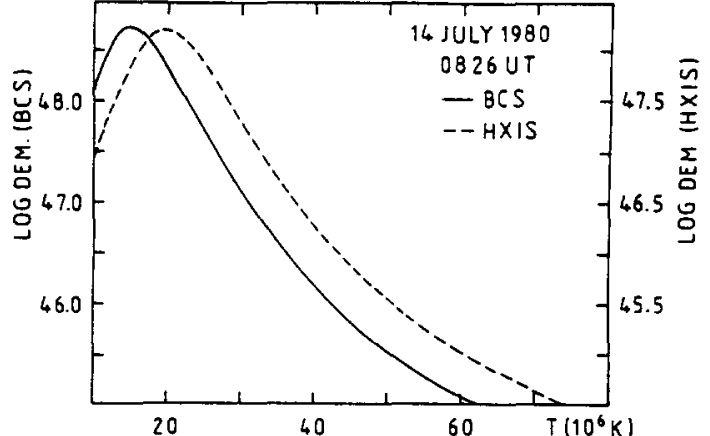

Fig. 1. An example of DEM distribution calculated from BCS data (solid line) and HXIS data (dashed line) separately.

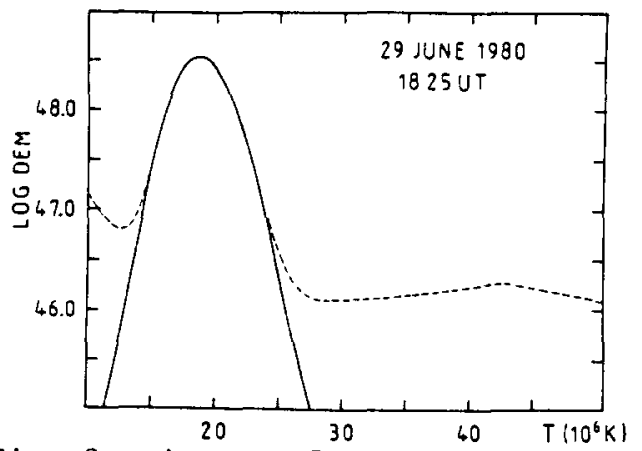

Fig. 2. An example of DEM distribution with a pronounced maximum near $\mathrm{T}=20 \mathrm{MK}$. The dashed line shows the estimated DEM error range as resulting from the random errors in the observational data.

\section{RESUITS}

Next, we have combined the BCS and HXIS data and calculated "final" DEM distributions from the whole set of data. In the case shown in Fig. 1 the final distribution is practically the same as the BCS distribution (solid line). In Figs. 2-4 some typical examples of the DEII distributions as obtained from BCS and HXIS data analysis are shown.

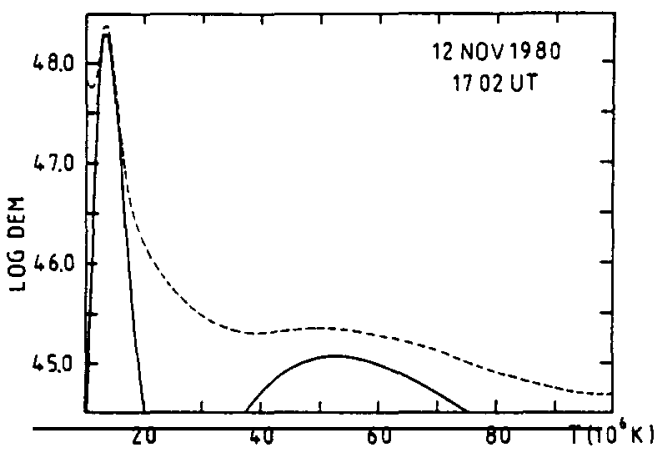

Fig. 3 .

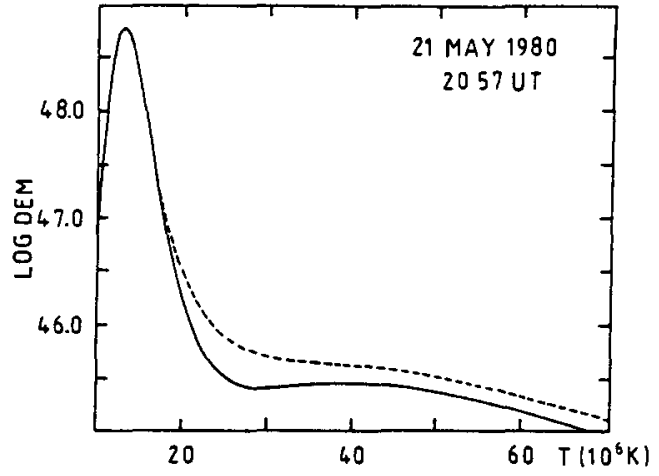

Fig. 4.

Examples of DEN distributions showing a high temperature (T $30 \mathrm{MK}$ ) component. The dashed line has the same meaning as in Fig. 2:

For some big flares, near the time of their highest $\mathrm{X}$-ray intensity, we have obtained pronounced maximum in the DEM distribution in the temperature range 15-25 MK (Fig. 2): We have carefuly tested such cases and have found that a flat DEM distribution cannot fit well the observations. Further tests of the reliability of such pronounced maxima in the DEM distribution are being continued.

Another important feature is that for some big flares we obtain a secondary high-temperature ( $T>30 \mathbb{M K}$ ) component in the DEM distribution during the time interval when the hard $X$-ray emission is strong ( $F i g .3$ and 4). This substantiates the results reported in $/ 8,9,10 /$ : The high-temperature component provides a thermal explanation of the fluxes recorded in the higher HXIS channels. Moreover, for some flares the component is separated (cf Fig. 3) from the typical DEM component ( $I \sim 15-20 \mathrm{IKK}$ ); this suggests that the hotter plasma is confined in a separate 1000 or region. When the hard $x$-rays decay, the high-tem- 
perature component quickly cools, i.e. shifts to the left in our diagrams.

Another characteristic feature of the model evolution during the flare development is that the DEM distributions are broader (i.e. the dispersion of the temperature is larger) during the flare heating, than during its cooling. This can be clearly shown by displaying the flare evolution in a two-temperature diagram, $\mathrm{T}_{\mathrm{Fe}}$ vs. $\mathrm{T}_{\mathrm{Ca}}$ (Fig. 5$)$.

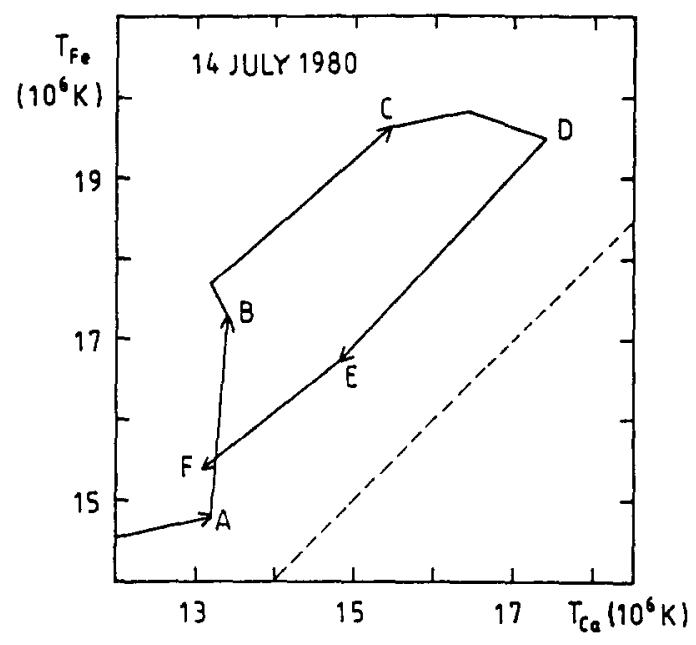

Fig. 5. An example of the flare evolution in the two-temperature diagram. See text for explanations. Characteristic time points are: A - 0823.8 UT, B - 0824.2 UT, C - 0825.4 UT, D - 0826.2 UT, E-0827.5 UT, F-0828.3 UT.

We see that at the beginning of the flare an impulsive heating occured ( $A B$ in Fig. 5) coinciding in time with the start of a strong increase in the hard $X-$ ray emission and this was followed by more gradual heating (BD). During the heating (AD) the temperature differences $\mathrm{T}_{\mathrm{Fe}}-\mathrm{T}_{\mathrm{Ca}}$ were systematically larger than during the cooling phase (DF).

In our DEM calculations we have also applied other iterative procedures (a generalized least-square method, a conjugate gradients method with various regularization assumptions) in order to investigate how strongly the obtained D IN distributions depend on the method. We have found that the distributions do not depend significantly on the choice of the fitting procedure.

The improvements in the DEM calculations here reported significantly increase the accuracy of the evaluation of the following integral flare parameters: the total emission measure, the radiation losses and the thermal energy content.

\section{AOKNOWLEDGENENTS}

This investigation has been carried out as the authons' contribution to the Uf SMII Workshop held in Oxford and funded by the Science and Engineering Research Council of the UK and by the Office of Naval Research of the US. J. J. and J.S. are thankful for the SERC financial support which allowed them to work several weeks at the MSSI. The authors thank Mr. S. Ratajczyk for drawinz the figures. 
REFERENCES

1. E. Antonucci, A.H. Gabriel, I.W. Acton, J.I. Culhane, J.G. Doyle, J.W. Leibacher, M.E. Machado, I:E: Orwig and C.G. Rapley, Solar Phys. 78, 107 (1982)

2. F. Bely-Dubau, J. Dubau, P. Faucher, A.H. Gabriel, M. Loulergue, I. Stenman-Clark, S. Volonte, E. Antonucci and C.G. Rapley, Mon. Not.R. Astr. SOC. 201, 1155 (1982)

3. J.R. Lemen, K.J.H. Phillips, R.D. Cowan, J. Hata and I.P. Grant, Innershell Transitions of Fe XXIII and Fe XXIV in the X-ray spectra of solar Flares, MSSI preprint (1983)

4. G.I. Withbroe, Solar Phys. 45, 301 (1975)

5. J. Sylwester, Thesis, Wroclaw University, (1977)

6. J.M. ShuIl and M. van Steenberg, Astrophys. J. Suppl. 48, 95 (1982)

7. HXIS Team, Predicted HXIS Counting rates, Utrecht, (JuIy 1983)

8. B. Sylwester, J. Jakimiec, J. Sylwester and B: Valnicek, Advo Space Res. 1, No. 13, 239 (1981)

9. P. Hoyng, A. Duijveman, M.E. Machado, D.M: Rust, Z. Svestka, A. Boelee, C. de Jager, K.J. Frost, H. Lafleur, G.M. Simnett, H.F. van Beek and B.E. Woodgate, Astrophys. J. Lett. 246, 1155 (1981)

10. M.E. Machado, A. Duijveman and B.R. Dennis, Solar Phys. 79, 85 (1982) 